\title{
The use of platelet rich plasma in the management of medication-related osteonecrosis of the jaws: a cohort study
}

Francesco Ricotta ${ }^{1}$, Carlotta Del Principe ${ }^{2}$, Agnese Ferri, Federico Bolognesi, Giovanni Leandro Arcangeli ${ }^{2}$, Roberto Parrulli $^{2}$, Pietro Felice ${ }^{2}$, Claudio Marchetti ${ }^{2}$

1 Azienda Ospedaliero-Universitaria di Bologna Policlinico S. Orsola-Malpighi

2 University of Bologna

Funding: The author(s) received no specific funding for this work.

Potential competing interests: The author(s) declared that no potential competing interests exist.

\section{Abstract}

Medication-related osteonecrosis of the jaw (MRONJ) is an adverse drug reaction that occurs in a very different ways and affects patients with complex medical histories.

The objective of this study is to evaluate the prognosis of surgically treated drug-related osteonecrotic lesions and to verify whether the platelet concentrates used during the surgical procedure can improve the prognosis.

The study was performed by analyzing a sample of 64 patients with medication-related osteonecrosis of the maxillary bones, in which 79 lesions were diagnosed and surgically treated at the S. Orsola-Malpighi Polyclinic in Bologna. The lesions treated with PRP showed a lower tendency to relapse compared to its non-use, the probability of recurrence.

In conclusion, this study demonstrates that treatment with PRP may have positive effects in the therapy of MedicationRelated Osteonecrosis of the Jaw (MRONJ).

Background: Medication-related osteonecrosis of the jaw (MRONJ) is an adverse drug reaction that occurs in a very different ways and affects patients with complex medical histories ${ }^{[1][2]}$. The symptoms and signs consist of pain, bone exposure, inflammation of the surrounding soft tissue swelling, and secondary infection or drainage ${ }^{[3]}$.

Decisions about treatment are based on factors such as age, sex, the stage of the disease, the severity of the BRONJ, the size and site of the lesion, exposure to drugs, and the presence of coexisting diseases ${ }^{[4]}$.

The objective of this study is to evaluate the prognosis of surgically treated drug-related osteonecrotic lesions and to verify whether the platelet concentrates used during the surgical procedure can improve the prognosis.

Materials and Methods: The study was performed by analyzing a sample of 64 patients with medication-related osteonecrosis of the maxillary bones, in which 79 lesions were diagnosed and surgically treated at the S. Orsola-Malpighi Polyclinic in Bologna. Subjects treated only with drug therapy and subjects with osteoradionecrosis were excluded.

The patients were then divided into two parallel cohorts: one made up of subjects treated using platelet concentrates, and one in which the patients were operated on without their use. Of these subjects, the relapse and the time in which it occurs 
was examined.

A descriptive analysis of the entire sample was performed, using the appropriate measures of central tendency and dispersion (mean and standard deviation) and frequency. The Cox mixed effects model to determine the hazard ratio $(\mathrm{HR})$ of relapse between the two cohorts, adding for the following confounding factors: age, sex, type of surgery, type of antiresorptive drug, diabetes and other metabolic disorders and AAOMS stage ${ }^{[5]}$.

Results: The average documented follow-up is 28 months and the antiresorptive drug taken were zoledronate in 43 patients (67\%), aledronate in 10 patients (15\%), ibandronate in 5 patients (8\%), risedronate in 5 patients $(5 \%)$ and denosumab in 3 patients (5\%).

Referring to the AAOMS staging system described in $2014^{[5]}$, of the 79 surgically treated lesions, 46 were diagnosed in stage $2(58 \%), 17$ in stage $3(22 \%)$ and 16 in stage $1(20 \%)$. Most of the lesions were located in the mandible $(75 \%)$. Of the 79 injuries affecting the maxillary bones, 62 (79\%) were treated with conservative surgery (26 treated with the help of PRP) and 16 (21\%) with resective surgery (7 of them treated with the help of PRP).

Of the 79 surgically treated lesions, 21 episodes $(27 \%)$ of relapse were documented.

In 33 cases platelet concentrates were used as adjuvant therapy to surgery, of these 8 relapsed (24\%) unlike 15 (76\%) who did not relapse. When they aren't used, relapses occurred in 13 cases (28\%).

In resective surgery (16 cases), PRP was associated in 7 cases, with 2 recurrence episodes (29\%). In the remaining 9 cases not associated with PRP, there were 2 relapses (22\%).

In the 62 conservative surgeries performed, platelet concentrates were used 26 times, with 5 relapses (20\%). In the remaining 36 cases, however, there were 11 relapses (31\%).

According to the 2014 AAOMS staging ${ }^{[5]}$ :

- Stage 1: 16 lesions, the use of platelet concentrates was implicated in 7 cases (no relapse episodes $0 \%$ ). In the 9 cases where they were not used, there were 2 recurrence episodes (22\%).

- Stage 2: 46 lesions, platelet concentrates were used 19 times with 7 relapse episodes (43\%) and 12 non-relapse episodes (57\%). While in the 27 cases of non-use, relapses were found in 7 cases $(26 \%)$.

- Stage 3: 17 lesions, platelet concentrates were implicated 7 times (1 relapse, 14\%). In the 10 remaining cases, there were 4 relapses (40\%), and 6 episodes without relapse (60\%).

From the results of the Cox model, it was highlighted how the risk of relapse for lesions treated with the aid of PRP was not statistically significant than the lesions treated without PRP (Hazard Ratio 0.81; $p=0.690$ ).

\section{Discussion:}

The treatment of Medication-Related Osteonecrosis of the Jaw (MRONJ) is much debated topic in the scientific community and numerous studies have been performed in this regard[6][7].

Today the surgical approach is not a secondary choice, but rather a primary therapeutic option even in the early stages of MRONJ, with the goal of stopping the progression of the disease in the most effective and early way possible. In fact, the conservative approach appears to be useful only in a limited number of cases and especially poorly effective in the advanced stages of MRONJ[8] [9]. 
Our study considered the treatment of 79 osteonecrotic lesions through resective surgery and conservative surgery, with or without the aid of platelet concentrates, finding 21 episodes (27\%) of relapses with an average follow-up of 28 months. Evaluating reviews of the literature available on MRONJ surgical therapy shows that most of clinical relapses occur within 6 months ${ }^{[10]}$, but it is important to underline that a significant number of relapses occur within 1 year of treatment, whatever the surgical therapy used ${ }^{[11]}$. Moreover many studies have a follow-up of 6 months or less, thus overestimating the treatment adopted.

For this reason it is important not only to monitor the patient but also to decree the success of the therapy only when mucosal healing is maintained in the absence of clinical and radiographic signs and symptoms 1 year after the completion of the surgical treatment.

Our study shows that the risk of relapse for lesions treated with the aid of platelet concentrates is lower than for lesions treated without (24\%vs28\%), that is confirmed by several studies documented in the literature ${ }^{[12][13]}$, but these results are not statistically significant $(p=0,690)$ and suggest that surgery has a fundamental role in the treatment of this pathology, of which PRP represents a valuable aid.

In fact PRP plays an important role in bone biology by releasing high amounts of growth factors, improving bone repair, stimulating angiogenesis and accelerating the healing. PRP appears to be a therapeutic choice in combination with antibiotic therapy and conventional surgery despite there is no definitive protocol for its use. Nevertheless, it is known in literature that therapeutic success depends on several factors such as the location of the lesion, the size of the lesion or the moment of diagnosis.

Indeed, our results did not demonstrate a true effectiveness of PRP in the treatment of this pathology.

More studies are needed to demonstrate the efficacy of this therapy.

There are no published scientific data to sufficiently support any specific treatment protocol, including the use of PRP together with surgical debridement, for the management of MRONJ ${ }^{[14][15]}$. Randomized controlled clinical trials of the use of PRP are needed.

\section{Conclusions:}

In the treatment of Medication-Related Osteonecrosis of the Jaw (MRONJ), the surgical approach certainly guarantees satisfactory success rates, as widely documented in the literature. This outcome seems to be confirmed by the positive effect of PRP in the treatment of these problems as an aid to the surgery itself. However, further studies are needed in this regard to confirm the effectiveness and establish a definitive use protocol.

\section{References}

1. `James R. Berenson, MD, Allan Lipton, MD. (1999). BISPHOSPHONATES IN THE TREATMENT OF MALIGNANT

BONE DISEASE. Annu. Rev. Med., vol. 50 (1), 237-248. doi:10.1146/annurev.med.50.1.237. 
2. 'Robert E Marx. (2003). Pamidronate (Aredia) and zoledronate (Zometa) induced avascular necrosis of the jaws: a growing epidemic. Journal of Oral and Maxillofacial Surgery, vol. 61 (9), 1115-1117. doi:10.1016/s02782391(03)00720-1.

3. 'Salvatore L. Ruggiero, John Fantasia, Eric Carlson. (2006). Bisphosphonate-related osteonecrosis of the jaw: background and guidelines for diagnosis, staging and management. Oral Surgery, Oral Medicine, Oral Pathology, Oral Radiology, and Endodontology, vol. 102 (4), 433-441. doi:10.1016/j.tripleo.2006.06.004.

4. 'D. Yoshiga, I. Nakamichi, Y. Yamashita, N. Yamamoto, et al. (2014). Prognosis factors in the treatment of bisphosphonate-related osteonecrosis of the jaw. Prognostic factors in the treatment of BRONJ. J Clin Exp Dent. doi:10.4317/jced.51213.

5. a, b, c Salvatore L. Ruggiero, Thomas B. Dodson, John Fantasia, Reginald Goodday, et al. (2014). American Association of Oral and Maxillofacial Surgeons Position Paper on Medication-Related Osteonecrosis of the Jaw-2014 Update. Journal of Oral and Maxillofacial Surgery, vol. 72 (10), 1938-1956. doi:10.1016/j.joms.2014.04.031.

6. 'D. Yoshiga, I. Nakamichi, Y. Yamashita, N. Yamamoto, et al. (2014). Prognosis factors in the treatment of bisphosphonate-related osteonecrosis of the jaw. Prognostic factors in the treatment of BRONJ. J Clin Exp Dent. doi:10.4317/jced.51213.

7. ^P.J. Voss, P. Poxleitner, R. Schmelzeisen, A. Stricker, et al. (2017). Update MRONJ and perspectives of its treatment. Journal of Stomatology, Oral and Maxillofacial Surgery, vol. 118 (4), 232-235. doi:10.1016/j.jormas.2017.06.012.

8. 'Morten Schiodt, Sven Otto, Stefano Fedele, Alberto Bedogni, et al. (2019). Workshop of European task force on medication-related osteonecrosis of the jaw-Current challenges. Oral Dis, vol. 25 (7), 1815-1821. doi:10.1111/odi.13160.

9. ^Francesco Ricotta, Salvatore Battaglia, Federico Bolognesi, Francesco Ceccariglia, et al. (2020). Use of CAD-CAM Bridging Mandibular Prosthesis in Osteonecrosis of the Jaw: The Experience of Our School. JCM, vol. 9 (11), 3516. doi:10.3390/jcm9113516.

10. 'Alberto Bedogni, Giorgia Saia, Giordana Bettini, Anita Tronchet, et al. (2011). Long-term outcomes of surgical resection of the jaws in cancer patients with bisphosphonate-related osteonecrosis. Oral Oncology, vol. 47 (5), 420 424. doi:10.1016/j.oraloncology.2011.02.024.

11. 'Athanassios Kyrgidis, Georgios Koloutsos, Konstantinos Vahtsevanos. (2009). Treatment protocols of bisphosphonate-related osteonecrosis of the jaws. Head Neck, vol. 31 (8), 1112-1113. doi:10.1002/hed.21148.

12. ' Coviello V et al. (2012). Platelet-rich plasma improves wound healing in multiple myeloma bisphosphonateassociated osteonecrosis of the jaw patients. Journal of Biological Regulators and Homeostatic Agents, vol. 26 .

13. 'Marcos Martins Curi, Giuliano Saraceni Issa Cossolin, Daniel Henrique Koga, Cristina Zardetto, et al. (2011). Bisphosphonate-Related Osteonecrosis of the Jaws-An Initial Case Series Report of Treatment Combining Partial Bone Resection and Autologous Platelet-Rich Plasma. Journal of Oral and Maxillofacial Surgery, vol. 69 (9), 24652472. doi:10.1016/j.joms.2011.02.078.

14. ^Pia Lopez-Jornet, Arturo Sanchez Perez, Rui Amaral Mendes, Aurelio Tobias. (2016). Medication-related osteonecrosis of the jaw: Is autologous platelet concentrate application effective for prevention and treatment? A systematic review. Journal of Cranio-Maxillofacial Surgery, vol. 44 (8), 1067-1072. doi:10.1016/j.jcms.2016.05.004. 
15. 'Leonzio Fortunato, Francesco Bennardo, Caterina Buffone, Amerigo Giudice. (2020). Is the application of platelet concentrates effective in the prevention and treatment of medication-related osteonecrosis of the jaw? A systematic review. Journal of Cranio-Maxillofacial Surgery, vol. 48 (3), 268-285. doi:10.1016/j.jcms.2020.01.014. 